\title{
Nuances in diagnosis and treatment of isolated brainstem tuberculomas
}

\author{
Nishanth Sadashiva $^{1} \cdot$ Bhagavatula Indira Devi $^{1}$
}

Received: 28 February 2017 / Accepted: 6 March 2017 / Published online: 14 March 2017

(C) Springer-Verlag Wien 2017

\section{Dear Editor,}

We read the comment by Beuy Joob and Viroj Wiwanitkit on our article "Isolated brainstem tuberculomas" [3] with great interest, and we acknowledge your interest and concern about this entity. In tropical countries a history of either systemic or neuro-tuberculosis may be supportive but not indicative of diagnosis. In such cases, if imaging is suggestive of tuberculoma, then antitubercular therapy (ATT) can be started with reasonable confidence without biopsy. In this sense, we completely agree with the authors that the pathological specimen is rarely required for confirmation of the diagnosis. In addition, as supported by our paper and confirmed by previous reports [1,2], the yield of a specimen for culture in cases of tuberculomas is rarely informative except to prove that it is an inflammatory lesion. Regarding misdiagnosing a tuberculoma as a malignant lesion, careful multimodality imaging can give a diagnosis in the majority of cases with reasonable confidence [4].

In cases where there is clinical deterioration in spite of antitubercular therapy (ATT), along with an increase in lesion size, a different diagnosis should be thought of, and stereotactic biopsy can be attempted. In cases where there is clinical improvement but the lesion size is not decreasing over time, then infection with drug-resistant

\section{Presentation at a conference NIL}

Clinical Trial Registration number NIL

Bhagavatula Indira Devi

bidevidr@gmail.com

1 Department of Neurosurgery, National Institute of Mental Health and Neurosciences, Neurosciences Faculty Building, Bangalore 560029, India tuberculosis can be considered and second-line treatment can be initiated. In our series, all patients who took ATT had clinical improvement but two showed slow resolution of the lesion on imaging despite being on ATT. These patients were suspected to be experiencing drug resistance, and second-line drugs were added, after which resolution occurred, although at a slower pace. We agree with the comments that change in ATT drugs should depend on the local prevalence of drug-resistant organisms, as biopsy may not yield any culture at all. Overall, biopsy should be considered when ATT fails to provide clinical improvement or there is radiological progress and there is strong suspicion of some other diagnosis.

\section{Compliance with ethical standards}

Funding No funding was received for this research.

\section{Conflict of interest None}

Ethical approval For this type of study formal consent is not required.

\section{References}

1. Ersahin M, Hakan T, Ayan E, Berkman Z, Ekinci O, Ceran N, Aker FV (2010) Diagnostic and therapeutic role of CT-guided stereotactic surgery in the management of intracranial tuberculomas. Turk Neurosurg 20:295-302

2. Rajshekhar V, Chandy MJ (1993) CT-guided stereotactic surgery in the management of intracranial tuberculomas. Br J Neurosurg 7:665671

3. Sadashiva N, Tiwari S, Shukla D, Bhat D, Saini J, Somanna S, Devi BI (2017) Isolated brainstem tuberculomas. Acta Neurochir (Wien)

4. Sonmez G, Ozturk E, Sildiroglu HO, Mutlu H, Cuce F, Senol MG, Kutlu A, Basekim CC, Kizilkaya E (2008) MRI findings of intracranial tuberculomas. Clin Imaging 32:88-92 